\title{
Kynurenine pathway in post-mortem prefrontal cortex and cerebellum in schizophrenia: relationship with monoamines and symptomatology
}

\author{
Amira Ben Afia ${ }^{1 \dagger}$, Èlia Vila ${ }^{2 \dagger}$, Karina S. MacDowell ${ }^{3,4}$, Aida Ormazabal ${ }^{5}$, Juan C. Leza ${ }^{3,4}$, Josep M. Haro ${ }^{4,6}$,
} Rafael Artuch $^{5}$, Belén Ramos $2,4,7^{* *}$ and Borja Garcia-Bueno ${ }^{3,4^{*}+}$ (D)

\begin{abstract}
Background: The cortico-cerebellar-thalamic-cortical circuit has been implicated in the emergence of psychotic symptoms in schizophrenia (SZ). The kynurenine pathway (KP) has been linked to alterations in glutamatergic and monoaminergic neurotransmission and to SZ symptomatology through the production of the metabolites quinolinic acid (QA) and kynurenic acid (KYNA).

Methods: This work describes alterations in KP in the post-mortem prefrontal cortex (PFC) and cerebellum (CB) of 15 chronic SZ patients and 14 control subjects in PFC and 13 control subjects in CB using immunoblot for protein levels and ELISA for interleukins and QA and KYNA determinations. Monoamine metabolites were analysed by high-performance liquid chromatography and SZ symptomatology was assessed by Positive and Negative Syndrome Scale (PANSS). The association of KP with inflammatory mediators, monoamine metabolism and SZ symptomatology was explored.

Results: In the PFC, the presence of the anti-inflammatory cytokine IL-10 together with IDO2 and KATII enzymes decreased in SZ, while TDO and KMO enzyme expression increased. A network interaction analysis showed that in the PFC IL-10 was coupled to the QA branch of the kynurenine pathway (TDO-KMO-QA), whereas IL-10 associated with KMO in CB. KYNA in the CB inversely correlated with negative and general PANSS psychopathology. Although there were no changes in monoamine metabolite content in the PFC in SZ, a network interaction analysis showed associations between dopamine and methoxyhydroxyphenylglycol degradation metabolite. Direct correlations were found between general PANSS psychopathology and the serotonin degradation metabolite, 5-hydroxyindoleacetic acid. Interestingly, KYNA in the CB inversely correlated with 5-hydroxyindoleacetic acid in the PFC.
\end{abstract}

\footnotetext{
*Correspondence: belen.ramos@sjd.es; bramos@fsjd.org;

bgbueno@med.ucm.es

${ }^{+}$Amira Ben Afia and Èlia Vila contributed equally to the work.

${ }^{+}$Belén Ramos and Borja Garcia-Bueno are joint senior authors.

${ }^{2}$ Psiquiatria Molecular, Parc Sanitari Sant Joan de Déu, Institut de Recerca

Sant Joan de Déu, Dr. Antoni Pujadas, 42, 08830 Sant Boi de Llobregat,

Barcelona, Spain

${ }^{3}$ Departamento de Farmacología y Toxicología, Facultad de Medicina,

Universidad Complutense de Madrid, Instituto de Investigación Sanitaria

Hospital 12 de Octubre (imas12), Instituto Universitario de Investigación en

Neuroquímica UCM, Avda. Complutense s/n, 28040 Madrid, Spain

Full list of author information is available at the end of the article
}

(c) The Author(s). 2021 Open Access This article is licensed under a Creative Commons Attribution 4.0 International License, which permits use, sharing, adaptation, distribution and reproduction in any medium or format, as long as you give appropriate credit to the original author(s) and the source, provide a link to the Creative Commons licence, and indicate if changes were made. The images or other third party material in this article are included in the article's Creative Commons licence, unless indicated otherwise in a credit line to the material. If material is not included in the article's Creative Commons licence and your intended use is not permitted by statutory regulation or exceeds the permitted use, you will need to obtain permission directly from the copyright holder. To view a copy of this licence, visit http://creativecommons.org/licenses/by/4.0/. The Creative Commons Public Domain Dedication waiver (http://creativecommons.org/publicdomain/zero/1.0/) applies to the data made available in this article, unless otherwise stated in a credit line to the data. 
Conclusions: Thus, this work found alterations in KP in two brain areas belonging to the cortico-cerebellar-thalamiccortical circuit associated with SZ symptomatology, with a possible impact across areas in 5-HT degradation.

Keywords: Schizophrenia, Cortico-cerebellar-thalamic-cortical circuit, Kynurenine pathway, 5-Hydroxyindoleacetic acid, Monoamine oxidase A, PANSS scale

\section{Background}

Schizophrenia (SZ) is a multifaceted psychiatric disorder in which dysfunction of particular neuronal circuits occurs. The cortico-cerebellar-thalamic-cortical circuit may contribute to negative symptomatology and cognitive deficits in SZ $[1,2]$. The cerebellum (CB) sends multiple inputs to the prefrontal cortex (PFC) through the thalamus, as well as dopaminergic inputs through the ventral tegmental area (VTA). There are feedback loops from the cortex to $\mathrm{CB}$ regulating the activity of PFC neurons. Thus, the prefrontal cortex is the end route of this circuit responsible for cognitive and negative symptoms in SZ, while the CB is a key area in this circuit integrating and modulating multiple inputs from cortical areas and responsible for sending output signals back to the same cortical region through the thalamus to correct errors in the cortical region. Recently, modulation of cerebellar activity has been proposed as a novel therapeutic intervention in SZ [3]. The control of PFC activity through cerebellar circuits improved symptoms, being of particular relevance for the negative ones [4]. However, the molecular basis underlying this improvement remains unknown.

Additionally, cognitive impairments have been particularly ascribed to the kynurenine pathway (KP) of tryptophan (Trp) metabolism [5]. Trp is commonly thought to be a precursor of the neurotransmitter $5-\mathrm{HT}$, but it can also be degraded to kynurenine (Kyn) [6] by one of these three enzymes, tryptophan 2,3-dioxygenase (TDO), indoleamine 2,3-dioxygenase 1 (IDO1), and the less studied indoleamine 2,3-dioxygenase 2 (IDO2) [7]. Next, the Kyn metabolism trifurcates into distinct branches. It can be converted by kynurenine aminotransferases (KATs IIV), mostly by KATII, to kynurenine acid (KYNA). It can also be oxidized by kynurenine 3-monooxygenase (KMO) to produce 3-hydroxykynurenine (3HK). Lastly, Kyn can undergo oxidative cleavage by kynureniase (KYNU) to form anthranilic acid (AA). Both 3HK and AA lead to the formation of quinolinic acid (QA) [8].

KYNA and QA are neuroactive compounds with purported links to neuropsychiatric diseases [9]. QA is an $\mathrm{N}$-methyl-D-aspartate receptor (NMDAR) agonist that can cause excitotoxicity, whereas KYNA is an NMDAR antagonist that can protect against excitotoxicity and apoptosis [10]. The KP in the brain is compartmentalized between different cellular types. IDOs are expressed in neurons, astrocytes and microglia; TDO is highly expressed in astrocytes and in some neurons; and KMO is predominantly expressed in microglia [11]. Thus, some authors have simplified the scenario, suggesting that astrocytes produce KYNA in the "hypoglutamatergic branch" of the KP, and microglia generates QA, responsible for the "neurotoxic branch" [11]. In SZ, increased brain KYNA may lead to excessive NMDAR blockade, which could trigger psychotic symptoms and cognitive deficits [12]. Apart from glutamate, these neuroactive metabolites can modulate other systems of neurotransmission, particularly those dependent on monoamines, such as dopamine (DA) and 5-HT [13]. Both neurotransmitters have been implicated in SZ symptoms $[14,15]$. Specifically, an excess of DA in subcortical areas and 5-HT hyperactivity at the serotonin $2 \mathrm{~A}$ receptors $(5-\mathrm{HT} 2 \mathrm{~A})$ on glutamate neurons in the cerebral cortex contribute to positive symptoms, while hypodopaminergic activity in the PFC is thought to cause negative symptoms [16, 17].

All of the elements of the KP are present in the brain and periphery [18] and regulated by pro-inflammatory cytokines, such as interleukin $1 \beta$ (IL-1 $\beta)$, interferon- $\gamma$ and tumour necrosis factor $\alpha$, which are considered main actors of neuro-immune interactions [19]. Among proinflammatory cytokines, IL- $1 \beta$ is the best described stimulatory cytokine of the KP in different inflammation-related conditions. IL-1 $\beta$ may induce the expression of the main enzymes of the KP, IDO1 and TDO2 in the brain [20]. Moreover, the study of anti-inflammatory cytokines such as IL-4 and IL-10 is growing, due to the current evidence supporting the existence of a dysregulated pro/anti-inflammatory cytokine balance in SZ [21]. In particular, IL10 levels were decreased in post-mortem brain PFC samples of SZ patients [22], and there are several studies suggesting the regulation of the KP by IL-10 and IL- 4 in neuropathological contexts [23, 24].

The dysregulation of the cytokine balance in SZ is associated to alterations on KP [24], and in particular, with the increased levels of KYNA observed at the central level [25]. However, the precise relationship between inflammation and KP, and whether these interactions could be implicated in the symptomatology of SZ remain partly unknown.

The aims of this study were (1) to investigate the possible alterations of the $\mathrm{KP}$ in brain $\mathrm{PFC}$ and $\mathrm{CB}$ in 
chronic SZ patients, (2) to explore the possible regulation of KP by pro/anti-inflammatory cytokines (IL-1 $\beta$, IL-10 and IL-4) and (3) to search for correlations between KP and monoamine metabolic pathways, and SZ symptomatology, in the last term.

\section{Methods}

\section{Post-mortem human brain samples}

Post-mortem human brain samples from the dorsolateral PFC (Brodmann area 9) and the cerebellum (CB; lateral cerebellar cortex) of patients with chronic schizophrenia (SZ; PCF, $n=15$; CB, $n=15$ ) and of control subjects with no history of psychiatric disorders (PFC, $n=14$; $\mathrm{CB}, n=13$ ) were obtained from the collection of Neurological Tissue of Sant Joan de Déu (SZ samples) [26] and the Brain Bank at the Hospital Universitari de Bellvitge (control samples). CB and PFC samples were matched from the same subjects except from one control in each cohort. This cohort was also used in a previous report that explored other inflammatory pathways in the same brain areas [26] with significant results. Based on these results, this cohort was selected for the study. Inclusion criteria for chronic SZ were (1) diagnosis of SZ spectrum, chronic type, following DSMIV criteria; (2) PANSS scale assessed pre-mortemly; (3) interval from symptom/neuropsychological evaluation to death $\leq 4$ years; and (4) age between 50 and 90 years and a duration of the illness longer than 25 years. Inclusion criteria for healthy controls will be (1) age and gender $( \pm$ $10 \%)$ similar to cases and (2) no history of mental disorder. Exclusion criteria for both control and patients are (1) the presence of neurological disorders or drug abuse, (2) accidental or natural cause of death that compromise the integrity of the brain region of interest, (3) the presence of a tumour or haemorrhages in the brain region of interest and (4) brain $\mathrm{pH}$ lower than 6. The demographic and clinical characteristics for these SZ subjects have been previously reported [26, 27] and are described in detail in Table S1 in the supplemental material. To control for the possible effect of antipsychotic treatment on the molecular measure, the last mean daily chlorpromazine equivalent dose was calculated for the antipsychotic treatment of each patient based on the electronic records of last drug prescriptions administered up to death (Table S1) as previously described in the general guidelines for clinically equivalent recommended doses of antipsychotics suggested in [28]. This so-called chlorpromazine equivalent dose provides a comparable standardized measure for each patient that allows for the comparison of different doses of antipsychotics among patients treated with different combinations of highly heterogeneous antipsychotic compounds. Given the heterogeneity of the treatment regimens of the patients in this study, a statistical comparison of different treatment regimens would not be feasible.

A possible tardive dyskinesia side effect of treatments was assessed in donors using the Abnormal Involuntary Movement Scale (AIMS) [29]. The total score was calculated using the sum of items 1 to 7 , which assess the severity of abnormal movements in different regions of the body. Each item was classified from 0 to 4 according to the severity ( 0 absence, 1 minimum, 2 mild, 3 moderate, 4 severe). The mean and standard deviation of the AIMS total score was $5.05 \pm 7.06(n=16)$ with a minimum value of 0 and a maximum value of $23.50 \%(n=8)$ of the subjects showed absent symptoms. $6.25 \%(n=1)$ of the subjects showed minimum severity of symptoms, 31.25\% $(n=5)$ mild, $6.25 \%(n=1)$ moderate and 6.25\% severe $(n=1)$. No subjects showed severe symptoms.

The study was approved by the Institutional Ethics Committee of Parc Sanitari Sant Joan de Déu. Written informed consent was obtained from each subject.

Specimens, extending from the pial surface to white matter and only including grey matter, were dissected and stored at $-80{ }^{\circ} \mathrm{C}$.

\section{Preparation of biological samples}

Tissue samples were homogenized by sonication in phosphate-buffered saline (PBS) mixed with a protease inhibitor cocktail (Complete ${ }^{\circ}$, Roche, Spain) $(\mathrm{pH}=7)$, followed by centrifugation at $12,000 \mathrm{~g}$ for $10 \mathrm{~min}$ at $4^{\circ} \mathrm{C}$.

\section{Cytokines and chemokine receptor levels}

IL-1 $\beta$ (Raybiotech, ELH-IL1b), IL-4 (Cusabio, CSBE04633h) and IL-10 (Raybiotech, ELH-IL10) tissue levels were detected using a commercially ELISA-based kit following the manufacturer's instructions. For IL1 $\beta$, the detection range is $0.3-100 \mathrm{pg} / \mathrm{ml}$, for IL10 $1-150 \mathrm{pg} / \mathrm{ml}$, and for IL4 $6.25-400 \mathrm{pg} / \mathrm{ml}$. The intra- and interassay CV were $<10 \%$ and $<12 \%$ for IL1 $\beta$ and IL10 and $<8 \%$ for IL4. Control values in PFC samples were $0.59 \pm 0.12$ (SD) $\mathrm{pg} / \mathrm{mg}$ of tissue for IL1 $\beta, 0.32 \pm 0.06 \mathrm{pg} / \mathrm{mg}$ of tissue for IL-10 and $7.34 \pm 1.19 \mathrm{pg} / \mathrm{mg}$ of tissue for IL4. Control values in CB samples were $0.57 \pm 0.06$ (SD) pg/ $\mathrm{mg}$ of tissue for IL1 $\beta, 0.47 \pm 0.06 \mathrm{pg} / \mathrm{mg}$ of tissue for IL10 and $8.44 \pm 1 \mathrm{pg} / \mathrm{mg}$ of tissue for IL4.

\section{Western blot analysis}

After determining and adjusting protein levels, homogenates were mixed with Laemmli sample buffer (Bio-Rad, USA), and $15 \mu \mathrm{g}$ was loaded into an electrophoresis gel. Once separated on the basis of molecular weight, proteins from the gels were blotted onto a nitrocellulose membrane using a semi-dry transfer system (Bio-Rad) and were incubated with specific primary antibodies against IDO1, IDO2, TDO; KMO, KATII and $\beta$-actin. 
Primary antibodies used were (1) indoleamine 2,3dioxygenase 1 (IDO1, 1:750 in BSA 1\%; sc25809, SCT); (2) indoleamine 2,3-dioxygenase 2 (IDO2, 1:750 in BSA 1\%; NBP2-44174, Novusbio); (3) tryptophan 2,3-dioxygenase 2 (TDO, 1:500 in BSA 1\%; ab123403, Abcam); (4) kynurenine 3-monooxygenase (KMO, 1:750 in BSA 1\%; NBP2-29936, Novusbio); (5) kynurenine aminotransferase 2 (KATII, 1:1000; sc377158, SCT); and (6) $\beta$-actin (1: 10000; A5441, Sigma).

Primary antibodies were recognized by the respective horseradish peroxidase-linked secondary antibodies. Blots were imaged using an Odyssey Fc System (Li-COR, Biosciences, Germany) and were quantified by densitometry (NIH ImageJ software). In all the WB analyses, the housekeeping $\beta$-actin was used as a loading control, and every western blot was performed at least three times in separate assays. The data were presented as percentage change from the control group.

\section{Kynurenine pathway metabolite measurement}

KYNA (Cloud-Clone, CED718Ge) and QA (CloudClone, CEK552Ge) tissue levels were detected using a commercially ELISA-based kit following the manufacturer's instructions. For KYNA, the detection range is $2.47-200 \mathrm{ng} / \mathrm{ml}$ and for QA $1.23-100 \mathrm{ng} / \mathrm{ml}$. The intraand interassay CV were $10 \%$ and $12 \%$ for KYNA and $\mathrm{QA}$, respectively. Control values in PFC samples were $808.64 \pm 76.3(\mathrm{SD}) \mathrm{pg} / \mathrm{mg}$ of tissue for KYNA and 2.74 $\pm 0.37 \mathrm{pg} / \mathrm{mg}$ of tissue for QA. Control values in $\mathrm{CB}$ samples were $1155.81 \pm 110.43(\mathrm{SD}) \mathrm{pg} / \mathrm{mg}$ of tissue for KYNA and $4.33 \pm 0.7 \mathrm{pg} / \mathrm{mg}$ of tissue for QA.

\section{Monoamines}

All samples were prepared in the same conditions. The minimum amount of brain tissue was $25 \mathrm{mg}$, which was homogenized in acidic buffer $(2 \mathrm{~mL}$ of 0.8 $\mathrm{mol} / \mathrm{L}$ perchloric acid, $40 \mu \mathrm{L}$ EDTA $0.1 \mathrm{mmol} / \mathrm{L}$ and $6 \mathrm{~mL}$ of ultrapure $\left.\mathrm{H}_{2} 0\right)$ at $4^{\circ} \mathrm{C}$, in a ratio $1: 9(1 \mathrm{mg}$ of tissue: $9 \mu \mathrm{L}$ of buffer). Samples were stored at $-80^{\circ} \mathrm{C}$ until analysis.

Then, after centrifugation $\left(1500 \mathrm{~g}, 10 \mathrm{~min}, 4^{\circ} \mathrm{C}\right)$, the supernatant was recovered and mixed 1:2 (v/V) with the chromatographic mobile phase $(0.1 \mathrm{~mol} / \mathrm{L}$ citrate/acetate buffer, $1.2 \mathrm{mmol} / \mathrm{L}$ EDTA/heptanesulphonic acid dissolved in $7 \%$ methanol: final $\mathrm{pH}=4$ ). The monoamines were measured by ion-pair HPLC coupled to electrochemical detection. This procedure was fully validated in our laboratory, as previously reported [30]. This procedure is accredited by the ISO 15189 norm (ENAC agency) and it is subjected to an external quality control scheme (www.ERNDIM.org).

\section{Network interaction analysis}

Interaction between the elements of KP and its relationship with pro/anti-inflammatory cytokines and monoamine metabolites were evaluated using a co-expression analysis adapted from a previous study in SZ [31]. This co-expression analysis for characterizing a pathway in a biological substrate is based on the assumption that proteins functionally related co-expressed [32]. Briefly, among all samples studied (control and SZ samples), only those with the highest (75th percentile) and lowest (25th percentile) fold-change values of the upstream member of the pathway were selected for analysis, starting from TDO, IDO1 and 2 (i.e. HighIDO1 group or Low-IDO1 group). Correlation analysis correlations with the downstream member of the pathway were performed. Differences in the protein expression levels of the downstream member between the group with samples with the highest protein levels and that with the lowest protein levels were evaluated. Significant correlations in this co-expression analysis indicate that the interaction distance between these two proteins is short in this network [33]. The pathway was "coupled" when all the downstream members of the pathway correlate with the upstream member, reflecting the proximity of the members in the pathway. The term "uncoupled pathway" was used when no significant correlations were detected between the downstream proteins and the upstream member in the pathway, indicating a higher distance between the elements of the pathway.

\section{Statistical analysis}

Quantitative values for protein or activity were tested for Gaussian distribution using the D'Agostino and Pearson omnibus normality test and compared using Student's unpaired $t$-test or Mann-Whitney test according to the distribution of each variable. Outliers were detected using the ROUT method for parametric variables with a significance $Q$ set at $1 \%$ for the detection and a Pierce test for the non-parametric variables. Spearman or Pearson correlation analyses were carried out according to the distribution of each variable to detect the association of the molecular measures with symptom severity. The false discovery rate (FDR) assessed with the Benjamini and Hochberg method was computed for all $p$ values resulting from comparisons with symptoms in each brain region and was set to 0.1 . Spearman or Pearson correlation analyses were also performed to assess the association of the molecular measures with and potential confounding factors (age, post-mortem delay, $\mathrm{pH}$, chlorpromazine dose (CPZd) and duration of illness). In case of finding a significant association with a potential confounder factor, a multiple linear regression model with a stepwise forward procedure was used to adjust to the co-variable where indicated. Statistical analyses were performed with GraphPad` Prism version 6.01 and SPSS 
24. All statistical tests were two-tailed and the significance level was set to 0.05 .

\section{Results}

Expression of pro/anti-inflammatory cytokines and KP elements in PFC and CB. Correlations with SZ symptomatology

IL-10 protein expression was significantly decreased in SZ subjects compared to control in the PFC (Fig. 1A). We did not find differences in IL-1 $\beta$ or IL-4 between groups (Fig. 1A, B).
We explored the branch of the KP composed by IDO-1 and 2 and KMO generating QA and the alternative branch composed by TDO and KATII that lead to KYNA production. The respective expression of both IDO enzymes decreased in the PFC of SZ subjects compared to control, especially for IDO-2 (Fig. 1D, E). TDO was increased in SZ while KAT II was markedly decreased (Fig. 1F, G) Contrarily, the expression of KMO was increased in SZ (Fig. 1H). Accordantly, QA tended to increase in SZ ( $p=$ 0.07) (Fig. 1J). However, no differences were found for KYNA content in the PFC (Fig. 1I).

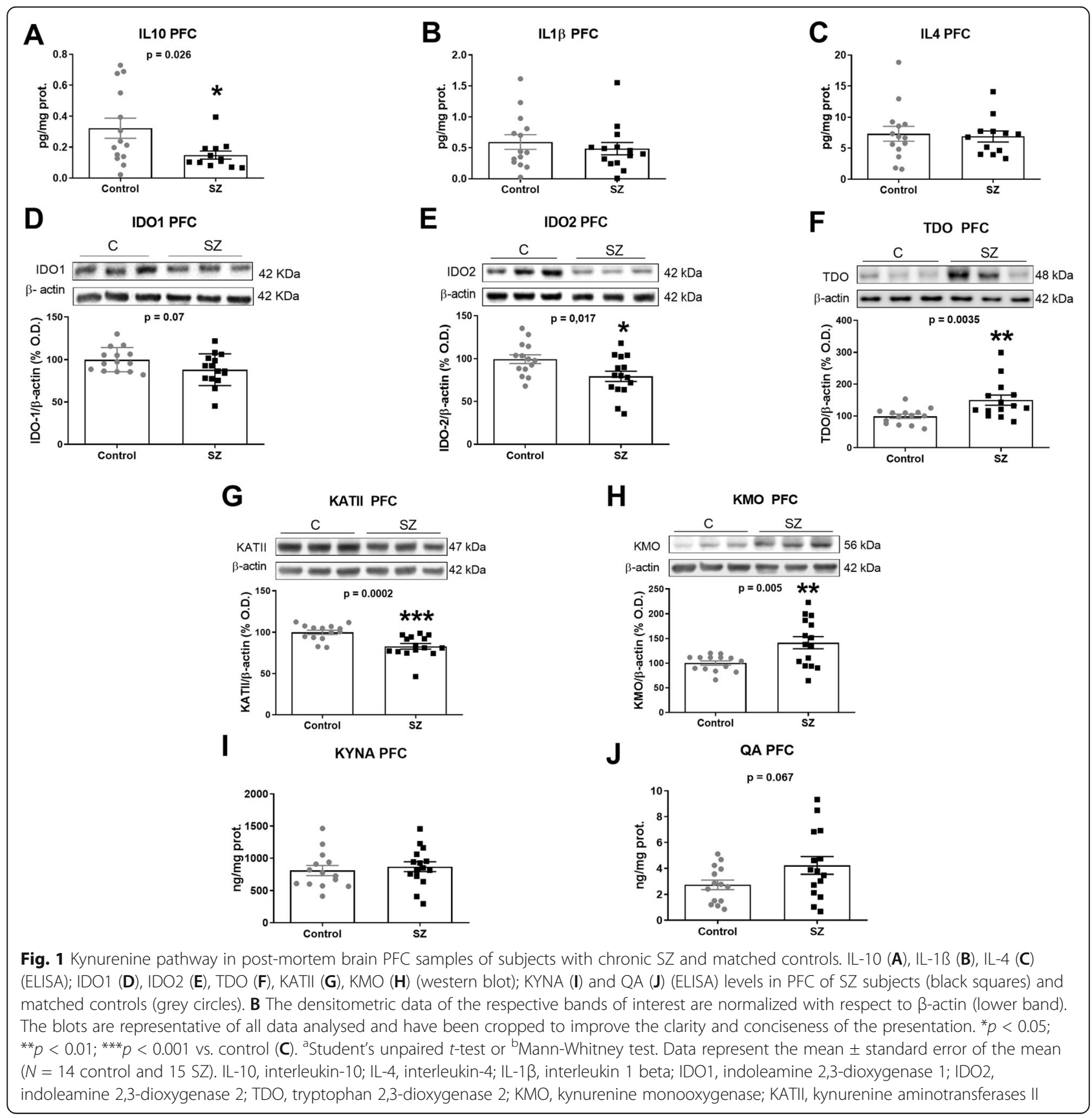


Table 1 Association analysis of other variables in the cohort

\begin{tabular}{llllll}
\hline $\begin{array}{l}\text { SZ-C cohort } \\
\text { Prefrontal cortex }(\boldsymbol{n}=\mathbf{2 9})\end{array}$ & Age & PMD & pH & $\begin{array}{l}\text { SZ cohort } \\
\text { Prefrontal cortex }(\boldsymbol{n}=\mathbf{1 5})\end{array}$ & CPZ \\
\hline IL10 & $-0.275^{2}$ & $0.228^{2}$ & $0.151^{2}$ & $0.263^{2}$ & $0.056^{2}$ \\
IDO2 & $-0.126^{1}$ & $0.283^{1}$ & $-0.130^{2}$ & $-0.177^{1}$ & $-0.154^{2}$ \\
TDO & $0.135^{2}$ & $\mathbf{0 . 3 9 8 ^ { 2 , a }}$ & $0.170^{2}$ & $-0.102^{1}$ & $-0.091^{2}$ \\
KATI & $-0.102^{1}$ & $0.231^{1}$ & $0.246^{2}$ & $0.216^{2}$ & $-\mathbf{0 . 5 9 1 ^ { 2 , a }}$ \\
KMO & $-0.187^{1}$ & $-0.006^{1}$ & $-0.295^{2}$ & $0.122^{1}$ & $-0.168^{2}$ \\
\hline
\end{tabular}

$S Z$, schizophrenia; $C$, control; $P M D$, post-mortem delay; $C P Z$, daily chlorpromazine dose; $D I$, duration of illness. Significant associations are indicated in bold ${ }^{a} p$ $<0.05$

${ }^{1}$ Pearson's $r$ is shown for parametric variables

${ }^{2}$ Spearman's $r$ is shown for non-parametric variables

Furthermore, we analysed the possible influence of other variables on the enzymes that significantly changed between groups (Table 1). The only significant correlation was found between TDO and post-mortem delay (PMD) (Table 1). Linear regression analysis revealed that the increase of TDO in SZ remained significant after adjusting by PMD $(\beta=0.456, p=0.015$, adjusted $R^{2}$ 0.244). To further explore the influence of antipsychotic medications and the duration of the illness on the enzymes altered in PFC, correlation analyses in the SZ group were performed. This analysis revealed no significant associations between $\mathrm{CPZ}$ dose and the levels of the enzymes and a significant inverse correlation between KATII and the duration of illness (Table 1).

We then explored whether KYNA and QA levels in the PFC correlate with the severity of the symptoms. KYNA did not correlate with negative symptoms in SZ patients $(r$ Pearson $=0.520$, uncorrected $p=0.046$, FDRadjusted $p=0.188)$.

The same inflammatory and KP-related parameters were measured in the $\mathrm{CB}$. There were no significant changes in any of the mediators measured (Fig. 2A-J).

Furthermore, an analysis of the co-expression of KP elements between $\mathrm{CB}$ and PFC in each individual revealed that IDO1 $(p$ value $=0.044 ; r=-0.565)$, TDO $(p$ value $=$ $0.017 ; r=0.646)$ and KMO ( $p=0.0006 ; r=0.818)$ were associated in controls, while none of them correlates between areas in the SZ group. These results suggest a possible different regulation of the expression of the elements of KP in each brain area in SZ and between control and SZ conditions.

We also analysed whether KYNA and QA levels in the $\mathrm{CB}$, respectively, correlate with the severity of the symptoms. Contrarily to the PFC, KYNA in the CB inversely correlated with both negative ( $r$ Pearson $=-0.734$, uncorrected $p=0.002$, FDR-adjusted $p=0.008)$ and general psychopathological subscales $(r$ Pearson $=-0.692$, uncorrected $p=0.004$, FDR-adjusted $p=0.008$ ) in SZ.

Moreover, we have analysed the possible influence of other variables on KYNA levels. Significant correlations were found between KYNA in the CB and CZPd.
A linear regression analysis revealed that the correlation between KYNA and both negative and general psychopathological PANSS remained significant after adjusting by CPZd (KYNA-Negative PANSS: $\beta=-1.157$, $p=0.001$, adjusted $R^{2}=0.662$; KYNA-General PANSS: $\beta=-0.787, p=0.032$, adjusted $R^{2}=0.485$ ).

\section{Network interaction analysis of the KP and cytokines}

A co-expression analysis (Fig. 3A, B) showed an inverse correlation between IL-10 and TDO in the PFC ( $r$ Pearson $=-0.801, p$ value $=0.003)$. Moreover, interactions between proteins showed significant associations between TDO and KMO $(r$ Pearson $=0.692, p$ value $=$ 0.009 ), and $\mathrm{KMO}$ and QA ( $r$ Spearman $=0.565, p$ value $=0.035$ ) (Fig. 3B). A comparison between the groups showed increased levels of KMO in individuals with higher levels of TDO compared to individuals with reduced TDO (TDO-KMO: $t$ test, $p$ value $=0.021$ ) (Figure S1A in Supplementary material). A tendency to associate is present between KMO and QA (KMO-QA: $t$ test, $p$ value $=0.06$ ) (Figure S1A in Supplementary material). These results indicate that IL-10 is acting upstream of TDO with the coupled TDO-KMO-QA pathway, which was altered in the PFC.

Our analysis also showed an interaction between IDO2 and KATII $(r$ Pearson $=0.642, p$ value $=0.018)$.

In the $\mathrm{CB}$, we found an inverse association of IL-1 $\beta$ with TDO $(r$ Spearman $=-0.836, p$ value $=0.001)$, and between TDO and KATII ( $r$ Pearson $=-0.603, p$ value $=$ 0.038) (Fig. 4A, B). KATII is uncoupled with its metabolite KYNA. Individuals with lower levels of IL-1 $\beta$ showed increased expression of TDO (IL-1 $\beta-T D O: ~ M a n n-$ Whitney $U, p$ value $=0.051$ ), and individuals with lower levels of TDO also expressed increased levels of KATII (TDO-KATII: Mann-Whitney $U, p$ value $=0.051)($ Figure S1B in Supplementary material). Furthermore, we found that IL-10 interacted downstream in the pathway, at the level of KMO ( $r$ Spearman $=-0.597, p$ value $=$ 0.031) (Figure S1A in Supplementary material). These results indicate that, contrary to the PFC, the TDO- 


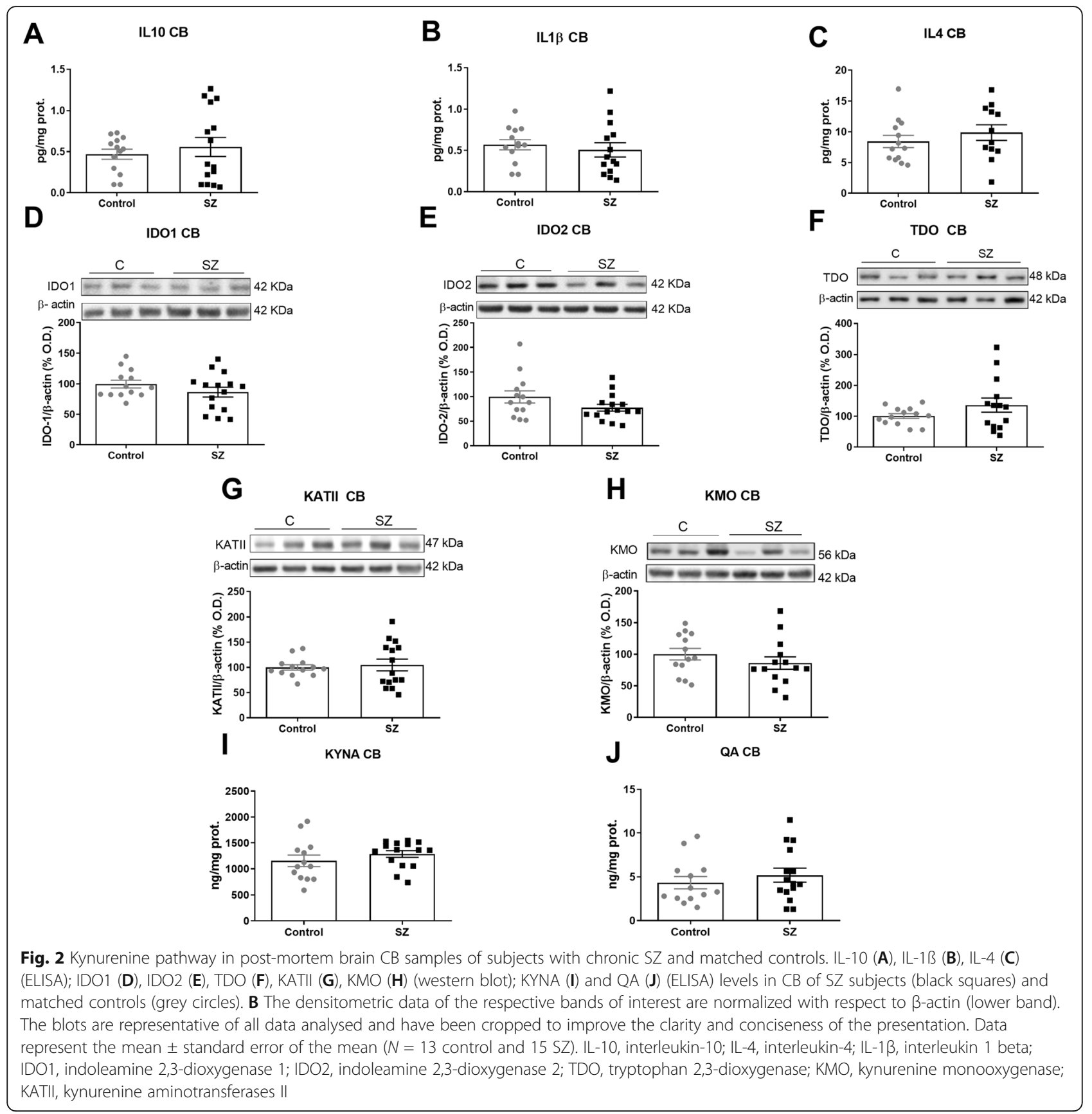

KMO-QA pathway is uncoupled in the CB, with IL-10 acting on KMO.

\section{Synthesis and metabolism of monoamines in PFC. Correlations with SZ symptomatology}

DA and 5-HT systems in the PFC were studied by measuring the levels of DA and homovanillic acid (HVA), and 5-hydroxyindoleacetic acid (5HIAA), respectively. Other metabolites such as 3-orthomethyldopa (3-OMD) and methoxyhydroxyphenylglycol (MHPG) for the DA pathway and 5-hydroxytryptophan (5HTP) for the 5-HT pathway were also tested [30].

There were no significant changes in any of the metabolites between groups (Fig. 5A).

We also investigate the existence of differences between control and SZ in the monoamine ratios for DA/ HVA and DA/MHPG. However, we did not find any significant differences (data not shown). Since the levels of 5-HT could not be detected in this approach, we further evaluate if there were significant differences in HVA/ HIAA ratio between control and SZ patients. We found 
A

B

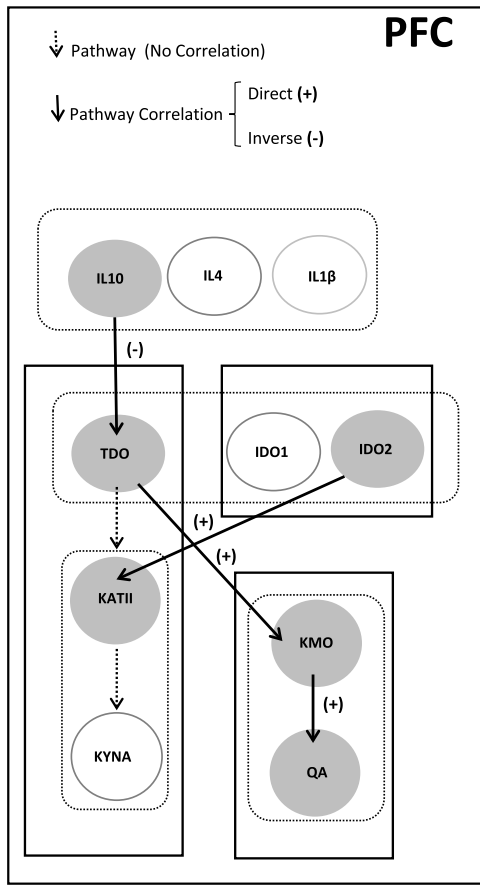

\begin{tabular}{|c|c|c|}
\hline \multicolumn{3}{|c|}{ CYTOKINES } \\
\hline Interaction & $\mathbf{R}$ & p value \\
\hline IL10-TDO & $-0.801^{1}$ & 0.003 \\
\hline |L10-IDO1 & $0.356^{1}$ & 0.255 \\
\hline |L10-IDO2 & $0.163^{1}$ & 0.430 \\
\hline IL10-KMO & $-0.396^{1}$ & 0.202 \\
\hline IL10-KATII & $0.242^{1}$ & 0.449 \\
\hline IL4-TDO & $0.168^{2}$ & 0.602 \\
\hline |L4-IDO1 & $-0.382^{1}$ & 0.220 \\
\hline |L4-IDO2 & $-0.335^{1}$ & 0.287 \\
\hline IL4-КMO & $-0.329^{2}$ & 0.297 \\
\hline IL4-KATII & $-0.244^{1}$ & 0.470 \\
\hline IL1 $\beta$-TDO & $0.066^{2}$ & 0.831 \\
\hline |L1ß-IDO1 & $0.170^{1}$ & 0.562 \\
\hline |L1及-IDO2 & $0.330^{1}$ & 0.249 \\
\hline 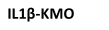 & $-0.060^{1}$ & 0.840 \\
\hline |L1ß-KATII & $0.188^{1}$ & 0.539 \\
\hline
\end{tabular}

\begin{tabular}{|lcc|}
\hline \multicolumn{3}{|c|}{ ENZYMES } \\
\hline Interaction & $\mathbf{R}$ & $\mathbf{p}$ value \\
\hline TDO-KMO & $\mathbf{0 . 6 9 2 ^ { 1 }}$ & $\mathbf{0 . 0 0 9}$ \\
\hline TDO-KATII & $-0.525^{1}$ & 0.080 \\
\hline IDO1 -KMO & $0.020^{1}$ & 0.946 \\
\hline IDO1-KATII & $0.162^{1}$ & 0.597 \\
\hline IDO2-KMO & $0.339^{1}$ & 0.236 \\
\hline IDO2-KATII & $\mathbf{0 . 6 4 2 ^ { 1 }}$ & $\mathbf{0 . 0 1 8}$ \\
\hline KMO-QA & $\mathbf{0 . 5 6 5 ^ { 2 }}$ & $\mathbf{0 . 0 3 5}$ \\
\hline KATII-KYNA & $-0.446^{2}$ & 0.110 \\
\hline 1Pearson's r correlation & \\
2Spearman's r correlation & \\
& & \\
& & \\
& & \\
\end{tabular}

Fig. 3 Co-expression analysis of the kynurenine pathway in the post-mortem prefrontal cortex. A The network model of the kynurenine pathway in the post-mortem prefrontal cortex. Samples with the highest and lowest expression levels were selected for the analysis. The white arrows show signal transduction between upstream and downstream elements. Black arrows indicate significant co-expression with the downstream element. Filled circles indicate a significant correlated or changed element in interaction analysis $(p<0.05)$. B Correlations using Pearson ${ }^{1}$ or Spearman ${ }^{2}$ analysis of the samples with the highest expression and lowest expression of upstream elements among all 29 samples were selected. Significant interactions are indicated with bold $(p<0.05)$

no significant differences in the SZ group compared to controls (Mann-Whitney test, $p$ value $=0.3101$ ). We further evaluate whether there were correlations between the levels of HVA and 5-HIAA in control and SZ conditions, respectively. We found a significant and direct correlation between both metabolites in the control group, but not in the SZ group (control: $r$ Pearson $=$ 0.874, $p=0.00004$; SZ: $r=0.483, p=0.068$ ).

Another network analysis revealed that DA levels inversely associated with MHPG from noradrenergic neurons ( $r$ Pearson $=-0.657, p=0.011)$, and showed a tendency to associate with HVA (Fig. 5B) $(r$ Pearson $=$ $-0.525, p=0.054$ ), suggesting that DA degradation pathways are coupled in the PFC. No other associations were found (Fig. 5B).

Moreover, we explored the possible association between DA levels and SZ symptoms. No significant correlation was found. We also explored the possible correlations between the expression of the degradation metabolites of monoamines in PFC with SZ symptoms. We found a significant correlation between PANSS general psychopathological subscale and the metabolite 5HIAA (degraded by monoamine oxidase A (MAO-A)) (Pearson $r=0.614$, uncorrected $p=0.015$; FDR-adjusted $p$ value $=0.090 ; p$ value threshold $=0.017)$ expression in PFC. A tendency to associate with PANSS general was also found for MHPG (substrate of MAO-A (Pearson $r$ $=-0.448, p=0.094)$ ). These results suggest a possible relationship between SZ symptoms and an increase activity of MAO-A in noradrenergic and serotoninergic neurons in the PFC.

\section{Correlations of the KP with monoamine pathway and SZ symptomatology}

We further explored the existence of correlations between KYNA in the CB and 5HIAA and MHPG in the PFC. We found an inverse correlation between KYNA in $\mathrm{CB}$ and 5HIAA in PFC ( $r$ Spearman $=-0.602, p=$ $0.023)$. These results suggest a possible modulation of 5HT degradation in the PFC mediated by KYNA inhibitory action on glutamatergic neurons in the CB (Fig. 5).

\section{Discussion}

We have found alterations in the KP in the corticocerebellar-thalamic-cortical circuit with a possible impact across areas in the metabolism of 5- $\mathrm{HT}$ and associated to general psychopathological symptom severity in SZ. 
A

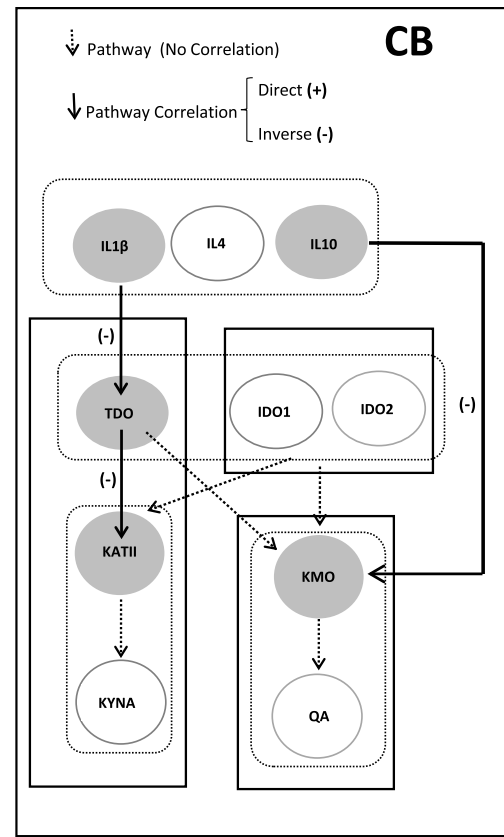

B

\begin{tabular}{|c|c|c|c|c|c|}
\hline \multicolumn{3}{|c|}{ CYTOKINES } & \multicolumn{3}{|c|}{ ENZYMES } \\
\hline Interaction & $\mathbf{R}$ & $p$ value & Interaction & $\mathbf{R}$ & $p$ value \\
\hline IL10-TD0 & $-0.308^{2}$ & 0.306 & TDO -КMO & $0.455^{1}$ & 0.137 \\
\hline IL10-IDO1 & $0.285^{2}$ & 0.324 & TDO-KATII & $-0.603^{1}$ & 0.038 \\
\hline |L10-IDO2 & $-0.104^{2}$ & 0.724 & IDO1-KMO & $0.045^{1}$ & 0.883 \\
\hline IL10-KMO & $-0.597^{2}$ & 0.031 & IDO1-KATII & $-0.057^{1}$ & 0.846 \\
\hline IL10-KATII & $0.373^{2}$ & 0.189 & IDO2-КMO & $0.482^{1}$ & 0.133 \\
\hline IL4-TDO & $-0.189^{2}$ & 0.557 & IDO2-KATII & $-0.477^{1}$ & 0.138 \\
\hline |L4-IDO1 & $0.261^{1}$ & 0.413 & KMO-QA & $-0.175^{2}$ & 0.587 \\
\hline IL4-IDO2 & $-0.410^{1}$ & 0.185 & KATII-KYNA & $0.320^{1}$ & 0.265 \\
\hline IL4-KMO & $-0.137^{1}$ & 0.671 & \multirow{7}{*}{\multicolumn{2}{|c|}{$\begin{array}{l}{ }^{1} \text { Pearson's r correlation } \\
{ }^{2} \text { Spearman's } r \text { correlation }\end{array}$}} & \\
\hline IL4-KATII & $0.167^{1}$ & 0.604 & & & \\
\hline IL1 $\beta$-TDO & $-0.836^{2}$ & 0.001 & & & \\
\hline IL1及-IDO1 & $-0.244^{1}$ & 0.470 & & & \\
\hline IL1及-IDO2 & $-0.411^{1}$ & 0.238 & & & \\
\hline І11в-кмо & $-0.498^{1}$ & 0.119 & & & \\
\hline IL1ß-KATII & $0.579^{1}$ & 0.062 & & & \\
\hline
\end{tabular}

Fig. 4 Co-expression analysis of kynurenine pathway in the post-mortem cerebellum. A The network model of the kynurenine pathway in the post-mortem cerebellum. Samples with the highest and lowest expression levels were selected for the analysis. The white arrows show signal transduction between upstream and downstream elements. Black arrows indicate significant co-expression with the downstream element. Filled circles indicate a significant correlated or changed element in interaction analysis $(p<0.05)$. B Correlations using Pearson ${ }^{1}$ or Spearman $^{2}$ analysis of the samples with the highest expression and lowest expression of upstream elements among all 28 samples were selected. Significant interactions are indicated with bold $(p<0.05)$

In our study, the changes observed in KP are exclusive of the PFC. Previous studies also focused on PFC reporting an increase in TDO expression and activity in SZ patients [25]. IDOs has been less explored: although one previous study did not found changes at the mRNA level [34], we have found a decreased protein expression in SZ. In the case of KATII and KMO, our results are opposite to previous ones in which KATII expression was increased or unchanged [25, 35], and KMO was decreased [35]. These changes in KATII and KMO were found at the mRNA level and in their enzymatic activity while we observed changes at the level of protein expression. Regarding KYNA and QA, we have only found a trend to increase in QA $(p=0.07)$, while others have focused on KYNA, reporting a consistent increase in the brain in SZ, PFC included [25, 36]. It is worth to mention that the expression of KATII and the levels of its metabolite KYNA do not seem to be related in PFC in our experimental conditions. This result may illustrate the complexity of KYNA production in the brain, suggesting the existence of possible alternative pathways, such as other enzymes and the implication of reactive oxygen or nitrogen species (ROS and RNS) in different in vitro and in vivo pathological scenarios [37, 38].
Our findings of increased expression of TDO in SZ in PFC suggest that the conversion of Trp to kynurenine is promoted probably in astrocytes. Moreover, the fact that TDO is coupled with increased KMO in SZ in PFC suggests a putative crosstalk between both cellular types. This raises the possibility that in SZ, kynurenine is produced by astrocytes, released to the extracellular fluid and transported into microglia cells through LAT1 or the leucine/arginine transporter Slc7a7 [39, 40]. This mechanism could compensate the decreased levels of IDO2 detected in PFC in our study, enhancing the production of "neurotoxic" QA by microglia in PFC. However, further studies are needed to explore the precise cellular types and associations involved.

There are no previous data for $\mathrm{KP}$ alterations in $\mathrm{CB}$ post-mortem samples in SZ to compare with our negative results, but the existence of functional alterations or in the expression at mRNA level/activity of the different elements of KP needs to be further explored.

The network interaction analysis made allowed us to explore possible interactions between the different elements of the KP and their regulatory mechanisms above their net expression levels.

Thus, our analysis suggested the role of IL-10 as an endogenous regulator of the KP in PFC and $\mathrm{CB}$, although at 


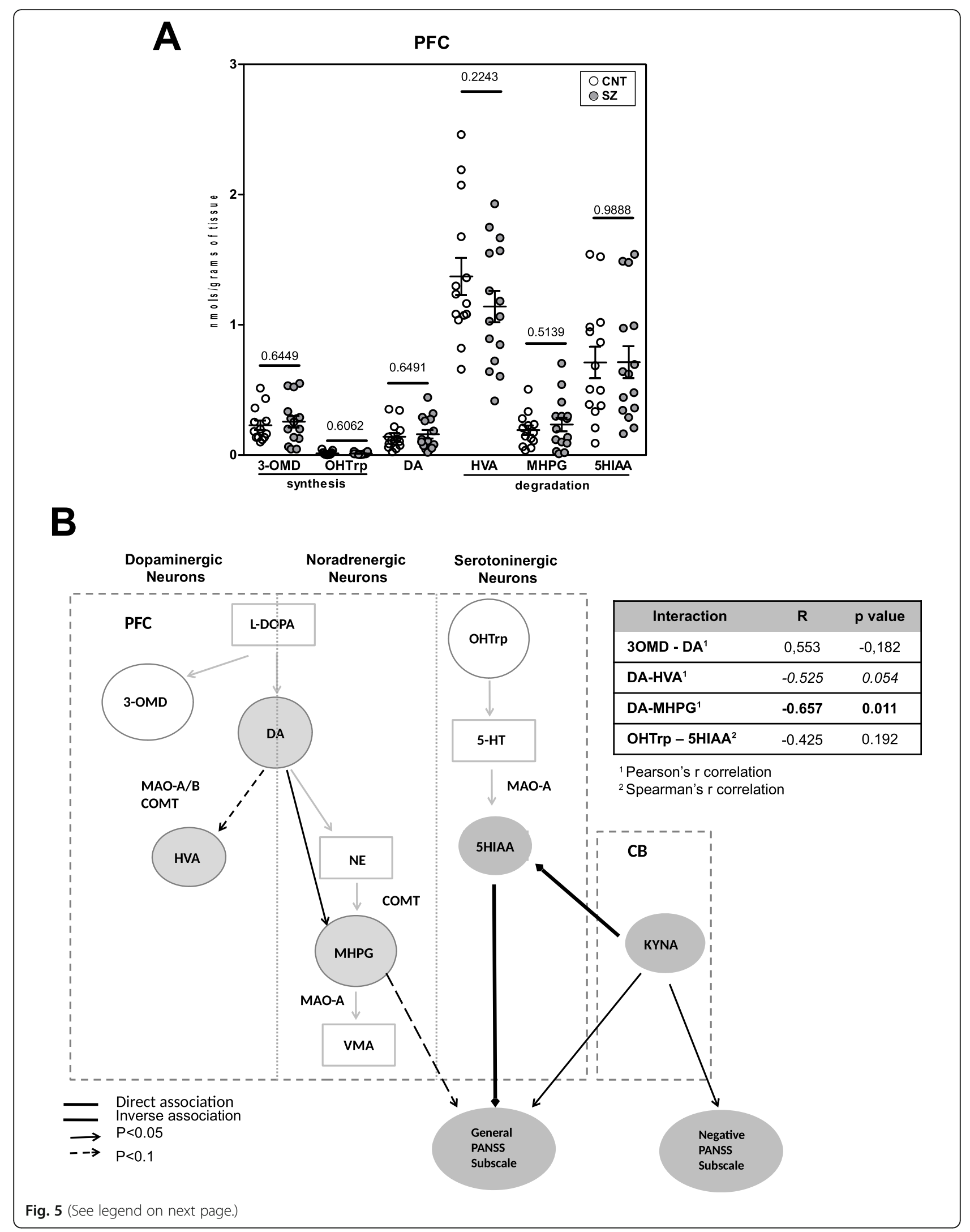




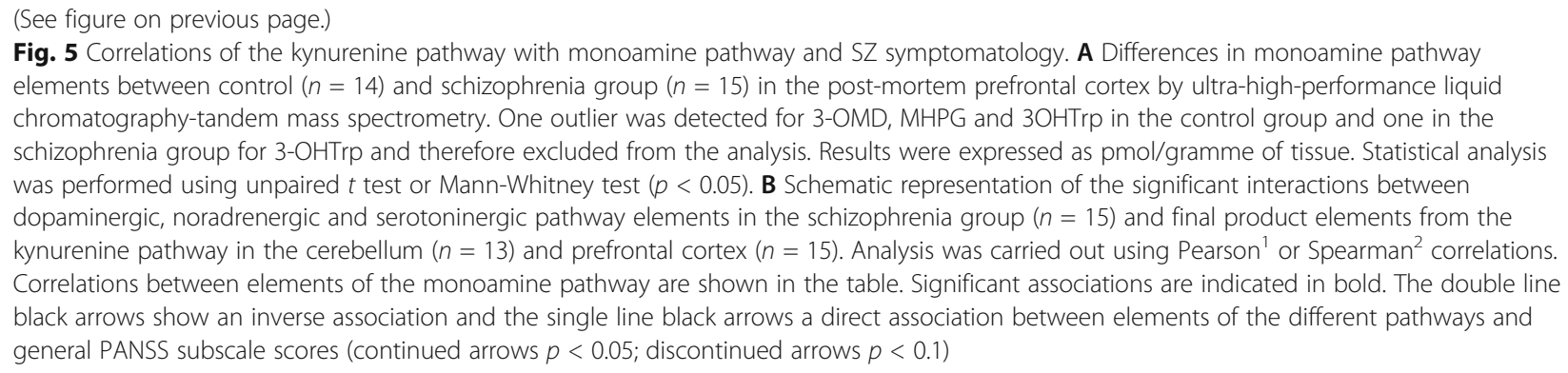

a different level. Decreased protein and mRNA expression of IL-10 in SZ has been also reported previously at the mRNA level in PFC, as well as no differences in IL-1 $\beta$, as in our experimental conditions [22, 33].

In our study, IL-10 is inversely related to the expression of TDO in PFC and KMO in $\mathrm{CB}$, respectively. A former study showed pro-inflammatory effects of QA via significant decreases of IL-10 at the systemic level in healthy volunteers [41]. The opposite relationship is also possible. Thus, IL-10 derived from $\mathrm{T}$ lymphocytes decreased IDO1 expression in the resolution phase of inflammation in the PFC of mice [23]. In our conditions, it remains to be elucidated whether the decreased levels of IL-10 in PFC observed are a consequence of the upregulation of coupled TDO to KMO and subsequent QA generation. In CB, IL-10 is also inversely associated with this microglial pathway, being coupled directly to KMO, and not related to TDO. No effect on QA was observed in $\mathrm{CB}$. The selective inhibition of microglial $\mathrm{KMO}$ is an emerging neuroprotective target [42].

Although we have not found changes in the expression levels of IL- $1 \beta$ in the two brain areas studied, the network interaction analysis in CB showed that IL- $1 \beta$ was inversely related to TDO $(p=0.001)$ and directly related with KATII, although in a lesser degree $(p=0.06)$. In our knowledge, there are no data about the relationship between IL-1 $\beta$ and KATII, but IL-1 $\beta$ has potential to induce KYNA in human astrocyte cultures increasing TDO expression, and also a strong correlation between IL-1 $\beta$ and TDO2 expression in human brain postmortem prefrontal grey matter from healthy donors was also shown [43]. Whether the discrepancies observed are due to the chronic nature of SZ in a possible compensatory mechanism between IL-1 $\beta$ and TDO remains to be elucidated, but it is worth to remark that the pathway IL-1 $\beta$-TDO-KATII is uncoupled to its final metabolite KYNA in CB, being the levels of KYNA similar to control samples.

Our correlation analysis points to KYNA as the main element of KP that correlated with SZ symptomatology. As commented above, previous evidences showed increased KYNA levels in SZ and in animal models of the disease $[25,35,36,44]$. This increase has been related to
SZ symptomatology (mainly in cognition) by means of alterations in glutamatergic, acetylcholinergic, GABAergic and dopaminergic neurotransmission [45-48]. However, there are no data about KYNA levels in CB, and their relation with SZ symptoms remains unexplored. It is worth to mention that some authors have found reduced levels of KYNA in the plasma of SZ patients [49]. Thus, all the previous evidence suggests that KYNA levels in SZ could vary between the brain and periphery, and in the different states and clinical subtypes of the disease, being affected by diverse confounding factors, such as the severity of inflammation or antipsychotics [36].

Although changes in monoamine degradation metabolites have been reported in CSF and plasma in SZ [50], our analysis did not reveal any change. Only a few studies have focused on monoamine metabolism in the postmortem brain, reporting an increase on DA or HVA in subcortical areas in SZ [51]. However, to our knowledge, no data were available for PFC in SZ.

The ratio HVA/5HIAA has been proposed as a biomarker for suicidal behaviour [52]. HVA/5HIAA ratio is highly constant in human CSF [53], and its alterations may reflect the existence of an imbalance between DA and 5-HT that could have pathological consequences. Interestingly, drug-free patients with SZ had significantly smaller correlations between CSF HVA and 5-HIAA than normal controls, and treatment with typical neuroleptics increased metabolite correlations [54]. In our study, we have detected a loss of correlation between HVA and 5-HIAA in SZ subjects. This evidence supports the relevance of the 5-HIAA metabolite in the study of SZ, but further studies should evaluate whether it is a cause or consequence of the disease.

There is an emerging interest of how modulating cerebellar activity induces changes in symptoms, being proposed that cortico-cerebellar-thalamic-cortical and the cerebellar-VTA-cortical circuits may underlie this effect by regulating PFC activity [3, 4]. We further explored the possibility that the levels of KYNA in CB could be related to monoamine metabolism in the PFC in the context of symptom severity in SZ. Since a glutamatergic/dopaminergic circuit has been recently identified between $\mathrm{CB}$ and PFC through the 
VTA, the most plausible hypothesis to explain cerebellar KYNA-related negative symptoms (associated with a hypodopaminergic activity in PFC) was effects on DA metabolites. Surprisingly, either DA or HVA in PFC correlated to KYNA cerebellar levels and negative symptoms.

However, we found a correlation between levels of KYNA in CB and 5-HIAA in PFC linked to general psychopathology symptoms. A trend to be associated with this subscale was also detected for MHPG. 5-HIAA and MHPG are the product and the substrate of the same enzyme, the monoamine oxidase A (MAO-A), respectively. This data raises the possibility that there may be increased MAO-A activity in serotoninergic and noradrenergic neurons in the PFC in subjects with more severe general psychopathological symptoms (affective, excitement and negative). We also found a direct interaction between HVA and 5-HIAA that agrees with the idea of increased MAO-A activity in PFC in SZ. Unfortunately, there are no previous studies exploring the activity of MAO-A in post-mortem brain PFC in SZ. This possible increased activity could result in a faster removal of 5-HT and noradrenaline neurotransmitters in this area linked to the severity in symptoms. This is in agreement with the proposed reduction of 5-HT in the brain in SZ responsible for emotional and affective symptoms [14]. However, there is not a consensus and other authors proposed an increase of 5-HT release, a hyperactive effect on 5-HT2A receptors on glutamatergic neurons in the cerebral cortex and a global higher serotoninergic tone in SZ [15].

5-HIAA and MHPG levels in plasma in SZ have been inversely and directly associated with depression/anxiety or excitement components of the PANSS scale, respectively [54], although some authors have alerted about the difficulty to correlate the levels of blood/urine monoamines with brain neurotransmission status, even in severe genetic conditions affecting DA and 5-HT metabolism [55]. However, the associations detected for these two metabolites with PANSS symptoms in our study are in the opposite direction. In agreement with our findings, 5-HIAA in CSF has also been reported to positively associate with several symptoms including some of the general psychopathological subscale [50]. In addition, previous studies have reported positive associations between 5-HIAA and KYNA in CSF and plasma in first-episode psychosis, which are the result of a global metabolism of different brain areas [56]. However, our findings showed an inverse correlation between KYNA levels in CB and 5-HIAA levels in the PFC suggesting a more complex interrelation between both brain areas. This connection might be mediated by glutamatergic outputs from CB that could be impacting on 5-HT degradation in the PFC. However, the neural bases of the implicated circuits are not clear. One possible complementary study of our pathway analysis could be to explore the relationship/s between pro/anti-inflammatory cytokines and monoamines and their metabolites in the SZ post-mortem brain in future studies.

Our study has some limitations: first, our cohort consisted of males only and it is needed to investigate possible gender differences. Second, although symptoms and in particular, negative symptoms, may be stable measures in chronic SZ patients [57], small variations in the clinical scores up to death may still slightly affect the associations we report here. Third, the patients with chronic SZ had long-term and heterogeneous antipsychotic medications. We have controlled the possible effect of antipsychotics on our findings using CPZd. This allowed us to identify an effect of antipsychotic treatment on KYNA levels in the CB; however, no associations were found for the significantly altered levels of the enzymes detected in PFC. A previous study has detected an influence of antipsychotics on cortical thickness and activity in PFC [58]. Since the kynurenine pathway could affect PFC activity, further studies are needed to completely rule out the effect of antipsychotic treatments on the changes in levels of KP in the PFC in SZ. Fifth, the SZ cohort in this study had a long duration of the illness which could be influencing the results. We controlled this variable and identify individuals with a longer duration of the disease who have lower levels of KATII in PFC suggesting a progressive reduction of this enzyme over the course of the disease. Sixth, the coexpression analysis is a prediction of how members of $\mathrm{KP}$ are functionally related among them and with monoamine metabolites. Future experimental interaction analyses with larger cohorts are needed to confirm these results. Seventh, a careful interpretation of our negative findings in CB in the SZ group compared to the control group should be done. Our analysis could be underpowered for detecting alterations in CB post-mortem samples and an analysis with larger groups will be needed.

\section{Conclusion}

Our study shows an interaction between active metabolites of the KP in the $\mathrm{CB}$ and 5-HT degradation in the PFC related to symptoms, offering a new insight of the molecular mechanism that underlined symptom severity in SZ.

\footnotetext{
Abbreviations

AA: Anthranilic acid; AIMS: Abnormal Involuntary Movement Scale; BSA: Bovine serum albumin; CB: Cerebellum; CSF: Cerebrospinal fluid; CPZd: Chlorpromazine dose; DA: Dopamine;

EDTA: Ethylenediaminetetraacetic acid; ELISA: Enzyme-linked immunosorbent assay; FDR: False discovery rate; $\mathrm{H}_{2} \mathrm{O}$ : Water; HPLC: High-performance liquid chromatography; HVA: Homovanillic acid; IDO: Indoleamine 2,3-dioxygenase; IL: Interleukin; KATs: Kynurenine aminotransferases; KMO: Kynurenine 3monooxygenase; KP: Kynurenine pathway; KYN: Kynurenine; KYNA: Kynurenic acid; KYNU: Kynureniase; MAO-A: Monoamine oxidase A; MHPG: Methoxyhydroxyphenylglycol; mg: Milligramme; ml: Mililitre;
} 
ng: Nanogramme; NMDAR: N-Methyl-D-aspartate receptor; LAT1: L-type/large neutral amino acid transporter 1; PANSS: Positive and negative syndrome scale; PBS: Phosphate-buffered saline; PFC: Post-mortem prefrontal cortex; pg: Pictogramme; PMD: Post-mortem delay; QA: Quinolinic acid; RNS: Reactive nitrogen species; ROS: Reactive oxygen species; SZ: Schizophrenia; TDO: Tryptophan 2,3-dioxygenase; Trp: Tryptophan; VTA: Ventral tegmental area; WB: Western blot; $\mu$ g: Microgramme; Il: Microlitre; 3HK: 3-Hydroxykynurenine; 3-OMD: 3-Orthomethyldopa; 5HIAA: 5-Hydroxyindoleacetic acid; 5-HT: Serotonin; 5-HTTP: 5-

Hydroxytryptophan; 5-HT2A: Serotonin 2A receptors

\section{Supplementary Information}

The online version contains supplementary material available at https://doi. org/10.1186/s12974-021-02260-6.

\section{Additional file 1. Material and Methods.}

\section{Acknowledgements}

Not applicable

\section{Authors' contributions}

$\mathrm{ABA}, \mathrm{EV}$, and $\mathrm{AO}$ contributed to the acquisition and analysis of the results; $\mathrm{KM}, J \mathrm{CL}, J \mathrm{JH}$, and RA contributed to the interpretation of the data for the work and revised critically for important intellectual content; and BR and BGB designed and drafted the work. The authors read and approved the final manuscript.

\section{Funding}

This work was supported by MINECO-FEDER Funds (SAF2016-75500-R) to JCL, by Miguel Servet grant (MS16/00153-CP16/00153) to BR financed and integrated into the National R + D + I and funded by the Instituto de Salud Carlos III (Spanish Ministry of Health) _ General Branch Evaluation and Promotion of Health Research-and the European Regional Development Fund (ERDF), by Predoctoral Fellowship Program from ISCIII (PFIS) FI19/00080 to EV and by CIBERSAM.

\section{Availability of data and materials}

The datasets used and/or analysed during the current study are available from the corresponding author on reasonable request.

\section{Declarations}

\section{Ethics approval and consent to participate}

The study was approved by the Institutional Ethics Committee of Parc Sanitari Sant Joan de Déu. Written informed consent was obtained from each subject.

\section{Consent for publication}

Not applicable

\section{Competing interests}

The authors declare that they have no competing interests.

\section{Author details}

${ }^{1}$ Laboratory of Genetics, Biodiversity and Bioresource Valorization, Higher Institute of Biotechnology of Monastir, University of Monastir, Monastir, Tunisia. ${ }^{2}$ Psiquiatria Molecular, Parc Sanitari Sant Joan de Déu, Institut de Recerca Sant Joan de Déu, Dr. Antoni Pujadas, 42, 08830 Sant Boi de Llobregat, Barcelona, Spain. ${ }^{3}$ Departamento de Farmacología y Toxicología, Facultad de Medicina, Universidad Complutense de Madrid, Instituto de Investigación Sanitaria Hospital 12 de Octubre (imas12), Instituto Universitario de Investigación en Neuroquímica UCM, Avda. Complutense s/n, 28040 Madrid, Spain. ${ }^{4}$ Centro de Investigación Biomédica en Red de Salud Mental, CIBERSAM (Biomedical Network Research Center of Mental Health), Ministry of Economy, Industry and Competitiveness Institute of Health Carlos III, Madrid, Spain. ${ }^{5}$ Clinical Chemistry Department, Institut de recerca Sant Joan de Déu and CIBERER-ISCIII, Passeig Sant Joan de Déu, 2. 08950, Esplugues de Llobregat, Barcelona, Spain. ${ }^{6}$ Parc Sanitari Sant Joan de Déu, Dr. Antoni Pujadas, 42, 08830 Sant Boi de Llobregat, Barcelona, Spain. ${ }^{7}$ Dept. de
Bioquímica i Biologia Molecular, Facultat de Medicina, Universitat Autònoma de Barcelona, Bellaterra, 08193 Barcelona, Spain.

Received: 18 January 2021 Accepted: 30 August 2021

Published online: 12 September 2021

\section{References}

1. Ho BC, Mola C, Andreasen NC. Cerebellar dysfunction in neuroleptic naive schizophrenia patients: clinical, cognitive, and neuroanatomic correlates of cerebellar neurologic signs. Biol Psychiatry. 2004;55(12):1146-53. https://doi. org/10.1016/j.biopsych.2004.02.020.

2. Barch DM. Cerebellar-thalamic connectivity in schizophrenia. Schizophr Bull. 2014;40(6):1200-3. https://doi.org/10.1093/schbul/sbu076.

3. Escelsior A, Belvederi Murri M. Modulation of cerebellar activity in schizophrenia: is it the time for clinical trials? Schizophr Bull. 2019:45(5):9479. https://doi.org/10.1093/schbul/sbz017.

4. Brady RO Jr, Gonsalvez I, Lee I, Ongur D, Seidman LJ, Schmahmann JD, et al. Cerebellar-prefrontal network connectivity and negative symptoms in schizophrenia. Am J Psychiatry. 2019;176(7):512-20. https://doi.org/10.1176/a ppi.ajp.2018.18040429.

5. Wonodi I, Schwarcz R. Cortical kynurenine pathway metabolism: a novel target for cognitive enhancement in schizophrenia. Schizophr Bull. 2010; 36(2):211-8. https://doi.org/10.1093/schbul/sbq002.

6. Thomas SR, Stocker $\mathrm{R}$. Redox reactions related to indoleamine 2,3dioxygenase and tryptophan metabolism along the kynurenine pathway. Redox Rep. 1999;4(5):199-220. https://doi.org/10.1179/1351 00099101534927

7. Fatokun AA, Hunt NH, Ball HJ. Indoleamine 2,3-dioxygenase 2 (IDO2) and the kynurenine pathway: characteristics and potential roles in health and disease. Amino Acids. 2013;45(6):1319-29. https://doi.org/10.1007/s00726013-1602-1.

8. Schwarcz R, Bruno JP, Muchowski PJ, Wu HQ. Kynurenines in the mammalian brain: when physiology meets pathology. Nat Rev Neurosci. 2012;13(7):465-77. https://doi.org/10.1038/nrn3257.

9. Schwarcz R, Pellicciari R. Manipulation of brain kynurenines: glial targets, neuronal effects, and clinical opportunities. J Pharmacol Exp Ther. 2002; 303(1):1-10. https://doi.org/10.1124/jpet.102.034439.

10. Muller N, Schwarz M. Schizophrenia as an inflammation-mediated dysbalance of glutamatergic neurotransmission. Neurotox Res. 2006;10(2): 131-48. https://doi.org/10.1007/BF03033242.

11. Parrott JM, Redus L, O'Connor JC. Kynurenine metabolic balance is disrupted in the hippocampus following peripheral lipopolysaccharide challenge. J Neuroinflammation. 2016;13(1):124. https://doi.org/10.1186/s12 974-016-0590-y.

12. Javitt DC, Zukin SR, Heresco-Levy U, Umbricht D. Has an angel shown the way? Etiological and therapeutic implications of the PCP/NMDA model of schizophrenia. Schizophr Bull. 2012;38(5):958-66. https://doi.org/10.1093/ schbul/sbs069.

13. Myint AM, Kim YK. Network beyond IDO in psychiatric disorders: revisiting neurodegeneration hypothesis. Prog Neuropsychopharmacol Biol Psychiatry. 2014;48:304-13. https://doi.org/10.1016/j.pnpbp.2013.08.008.

14. Jayamohananan H, Manoj Kumar MK. T PA: 5-HIAA as a potential biological marker for neurological and psychiatric disorders. Adv Pharm Bull. 2019;9(3): 374-81. https://doi.org/10.15171/apb.2019.044.

15. Stahl SM. Beyond the dopamine hypothesis of schizophrenia to three neural networks of psychosis: dopamine, serotonin, and glutamate. CNS Spectr. 2018;23(3):187-91. https://doi.org/10.1017/S1092852918001013.

16. Kesby JP, Eyles DW, McGrath JJ, Scott JG. Dopamine, psychosis and schizophrenia: the widening gap between basic and clinical neuroscience. Transl Psychiatry. 2018;8(1):30. https://doi.org/10.1038/s41398-017-0071-9.

17. Toda M, Abi-Dargham A. Dopamine hypothesis of schizophrenia: making sense of it all. Curr Psychiatry Rep. 2007;9(4):329-36. https://doi.org/10.1007/ s11920-007-0041-7.

18. Schwarcz R, Stone TW. The kynurenine pathway and the brain: challenges, controversies and promises. Neuropharmacology. 2017;112(Pt B):237-47. https://doi.org/10.1016/j.neuropharm.2016.08.003.

19. Miller $\mathrm{AH}$, Haroon $\mathrm{E}$, Raison $\mathrm{CL}$, Felger JC. Cytokine targets in the brain: impact on neurotransmitters and neurocircuits. Depress Anxiety. 2013;30(4): 297-306. https://doi.org/10.1002/da.22084.

20. Zunszain PA, Anacker C, Cattaneo A, Choudhury S, Musaelyan K, Myint AM, et al. Interleukin-1 beta: a new regulator of the kynurenine pathway affecting 
human hippocampal neurogenesis. Neuropsychopharmacology. 2012;37(4): 939-49. https://doi.org/10.1038/npp.2011.277.

21. Momtazmanesh S, Zare-Shahabadi A, Rezaei N. Cytokine alterations in schizophrenia: an updated review. Front Psychiatry. 2019;10:892. https://doi. org/10.3389/fpsyt.2019.00892.

22. Pandey GN, Rizavi HS, Zhang $H$, Ren X. Abnormal gene and protein expression of inflammatory cytokines in the postmortem brain of schizophrenia patients. Schizophr Res. 2018;192:247-54. https://doi.org/10.1 016/j.schres.2017.04.043.

23. Laumet G, Edralin JD, Chiang AC, Dantzer R, Heijnen CJ, Kavelaars A. Resolution of inflammation-induced depression requires T lymphocytes and endogenous brain interleukin-10 signaling. Neuropsychopharmacology. 2018;43(13):2597-605. https://doi.org/10.1038/s41386-018-0154-1.

24. Pedraz-Petrozzi B, Elyamany O, Rummel C, Mulert C. Effects of inflammation on the kynurenine pathway in schizophrenia - a systematic review. J Neuroinflammation. 2020;17(1):56. https://doi.org/1 0.1186/s12974-020-1721-z.

25. Kindler J, Lim CK, Weickert CS, Boerrigter D, Galletly C, Liu D, et al. Dysregulation of kynurenine metabolism is related to proinflammatory cytokines, attention, and prefrontal cortex volume in schizophrenia. Mol Psychiatry. 2020;25(11):2860-72.

26. MacDowell KS, Pinacho R, Leza JC, Costa J, Ramos B, Garcia-Bueno B. Differential regulation of the TLR4 signalling pathway in post-mortem prefrontal cortex and cerebellum in chronic schizophrenia: relationship with SP transcription factors. Prog Neuropsychopharmacol Biol Psychiatry. 2017; 79(Pt B):481-92. https://doi.org/10.1016/.jpnpbp.2017.08.005.

27. Pinacho R, Villalmanzo N, Roca M, Iniesta R, Monje A, Haro JM, et al. Analysis of Sp transcription factors in the postmortem brain of chronic schizophrenia: a pilot study of relationship to negative symptoms. J Psychiatr Res. 2013;47(7):926-34. https://doi.org/10.1016/j.jpsychires.2013.03. 004.

28. Gardner DM, Murphy AL, O'Donnell H, Centorrino F, Baldessarini RJ. International consensus study of antipsychotic dosing. Am J Psychiatry. 2010;167(6):686-93. https://doi.org/10.1176/appi.ajp.2009.09060802.

29. Burti L, Parolin A, Zanotelli R. Tardive dyskinesia. AlMS (Abnormal Involuntary Movement Scale) as a diagnostic and research tool. Minerva Med. 1981;72(42):2829-36.

30. Batllori M, Molero-Luis M, Ormazabal A, Casado M, Sierra C, Garcia-Cazorla A, et al. Analysis of human cerebrospinal fluid monoamines and their cofactors by HPLC. Nat Protoc. 2017;12(11):2359-75. https://doi.org/10.1038/nprot.201 7.103.

31. Hirayama-Kurogi M, Takizawa Y, Kunii Y, Matsumoto J, Wada A, Hino M, et al. Downregulation of GNA13-ERK network in prefrontal cortex of schizophrenia brain identified by combined focused and targeted quantitative proteomics. J Proteomics. 2017;158:31-42. https://doi.org/10.1 016/j.jprot.2017.02.009.

32. Stuart JM, Segal E, Koller D, Kim SK. A gene-coexpression network for global discovery of conserved genetic modules. Science. 2003;302(5643):249-55. https://doi.org/10.1126/science.1087447.

33. Lopez-Gonzalez I, Pinacho R, Vila E, Escanilla A, Ferrer I, Ramos B. Neuroinflammation in the dorsolateral prefrontal cortex in elderly chronic schizophrenia. Eur Neuropsychopharmacol. 2019;29(3):384-96. https://doi. org/10.1016/j.euroneuro.2018.12.011.

34. Miller CL, Llenos IC, Dulay JR, Barillo MM, Yolken RH, Weis S. Expression of the kynurenine pathway enzyme tryptophan 2,3-dioxygenase is increased in the frontal cortex of individuals with schizophrenia. Neurobiol Dis. 2004; 15(3):618-29. https://doi.org/10.1016/.nbd.2003.12.015.

35. Sathyasaikumar KV, Stachowski EK, Wonodi I, Roberts RC, Rassoulpour A, McMahon RP, et al. Impaired kynurenine pathway metabolism in the prefrontal cortex of individuals with schizophrenia. Schizophr Bull. 2011; 37(6):1147-56. https://doi.org/10.1093/schbul/sbq112.

36. Plitman E, Iwata Y, Caravaggio F, Nakajima S, Chung JK, Gerretsen P, et al. Kynurenic acid in schizophrenia: a systematic review and metaanalysis. Schizophr Bull. 2017;43(4):764-77. https://doi.org/10.1093/ schbul/sbw221.

37. Blanco Ayala T, Lugo Huitron R, Carmona Aparicio L, Ramirez Ortega D, Gonzalez Esquivel D, Pedraza Chaverri J, et al. Alternative kynurenic acid synthesis routes studied in the rat cerebellum. Front Cell Neurosci. 2015;9: 178. https://doi.org/10.3389/fncel.2015.00178.

38. Ramos-Chavez LA, Lugo Huitron R, Gonzalez Esquivel D, Pineda B, Rios C, Silva-Adaya $D$, et al. Relevance of alternative routes of kynurenic acid production in the brain. Oxid Med Cell Longev. 2018;2018:5272741-14. https://doi.org/10.1155/2018/5272741

39. Huttunen J, Peltokangas S, Gynther M, Natunen T, Hiltunen M, Auriola S, et al. L-type amino acid transporter 1 (LAT1/Lat1)-utilizing prodrugs can improve the delivery of drugs into neurons, astrocytes and microglia. Sci Rep. 2019;9(1):12860. https://doi.org/10.1038/s41598-019-49009-z.

40. Rossi F, Casano AM, Henke K, Richter K, Peri F. The SLC7A7 transporter identifies microglial precursors prior to entry into the brain. Cell Rep. 2015: 11(7):1008-17. https://doi.org/10.1016/j.celrep.2015.04.028.

41. Maes M, Mihaylova I, Ruyter MD, Kubera M, Bosmans E. The immune effects of TRYCATs (tryptophan catabolites along the IDO pathway): relevance for depression - and other conditions characterized by tryptophan depletion induced by inflammation. Neuro Endocrinol Lett. 2007;28(6):826-31.

42. Parrott JM, O'Connor JC. Kynurenine 3-monooxygenase: an influential mediator of neuropathology. Front Psychiatry. 2015;6:116. https://doi.org/1 0.3389/fpsyt.2015.00116.

43. Sellgren CM, Kegel ME, Bergen SE, Ekman CJ, Olsson S, Larsson M, et al. A genome-wide association study of kynurenic acid in cerebrospinal fluid: implications for psychosis and cognitive impairment in bipolar disorder. Mol Psychiatry. 2016;21(10):1342-50. https://doi.org/10.1038/mp.2015.186.

44. Huang X, Ding W, Wu F, Zhou S, Deng S, Ning Y. Increased plasma kynurenic acid levels are associated with impaired attention/vigilance and social cognition in patients with schizophrenia. Neuropsychiatr Dis Treat. 2020;16:263-71. https://doi.org/10.2147/NDT.S239763.

45. Fujigaki H, Mouri A, Yamamoto Y, Nabeshima T, Saito K. Linking phencyclidine intoxication to the tryptophan-kynurenine pathway: therapeutic implications for schizophrenia. Neurochem Int. 2019;125:1-6. https://doi.org/10.1016/j.neuint.2019.02.001.

46. Tufvesson-Alm M, Schwieler L, Schwarcz R, Goiny M, Erhardt S, Engberg G. Importance of kynurenine 3-monooxygenase for spontaneous firing and pharmacological responses of midbrain dopamine neurons: relevance for schizophrenia. Neuropharmacology. 2018;138:130-9. https://doi.org/10.1016/ j.neuropharm.2018.06.003.

47. Kegel ME, Johansson V, Wetterberg L, Bhat M, Schwieler L, Cannon TD, et al. Kynurenic acid and psychotic symptoms and personality traits in twins with psychiatric morbidity. Psychiatry Res. 2017;247:105-12. https://doi.org/10.101 6/j.psychres.2016.11.017.

48. Beggiato $S$, Tanganelli $S$, Fuxe $K$, Antonelli T, Schwarcz R, Ferraro L. Endogenous kynurenic acid regulates extracellular GABA levels in the rat prefrontal cortex. Neuropharmacology. 2014;82:11-8. https://doi.org/10.101 6/j.neuropharm.2014.02.019.

49. Chiappelli J, Notarangelo FM, Pocivavsek A, Thomas MAR, Rowland LM, Schwarcz R, et al. Influence of plasma cytokines on kynurenine and kynurenic acid in schizophrenia. Neuropsychopharmacology. 2018;43(8): 1675-80. https://doi.org/10.1038/s41386-018-0038-4.

50. Davison J, O'Gorman A, Brennan L, Cotter DR. A systematic review of metabolite biomarkers of schizophrenia. Schizophr Res. 2018;195:32-50. https://doi.org/10.1016/j.schres.2017.09.021.

51. Toru M, Nishikawa T, Mataga N, Takashima M. Dopamine metabolism increases in post-mortem schizophrenic basal ganglia. J Neural Transm. 1982;54(3-4):181-91. https://doi.org/10.1007/BF01254928.

52. Jokinen J, Nordstrom AL, Nordstrom P. The relationship between CSF HVA/ 5-HIAA ratio and suicide intent in suicide attempters. Arch Suicide Res. 2007;11(2):187-92. https://doi.org/10.1080/13811110701250093.

53. Geracioti TD Jr, Keck PE Jr, Ekhator NN, West SA, Baker DG, Hill KK, et al. Continuous covariability of dopamine and serotonin metabolites in human cerebrospinal fluid. Biol Psychiatry. 1998;44(3):228-33. https://doi.org/10.101 6/50006-3223(97)90372-3.

54. Watanabe K, Miura I, Kanno-Nozaki K, Horikoshi S, Mashiko H, Niwa S, et al. Associations between five-factor model of the Positive and Negative Syndrome Scale and plasma levels of monoamine metabolite in patients with schizophrenia. Psychiatry Res. 2015;230(2):419-23. https://doi.org/10.1 016/j.psychres.2015.09.030.

55. Batllori M, Molero-Luis M, Casado M, Sierra C, Artuch R, Ormazabal A. Biochemical analyses of cerebrospinal fluid for the diagnosis of neurometabolic conditions. What can we expect? Semin Pediatr Neurol. 2016;23(4):273-84. https://doi.org/10.1016/j.spen.2016.11.002.

56. Yao JK, Dougherty GG Jr, Reddy RD, Keshavan MS, Montrose DM, Matson WR, et al. Altered interactions of tryptophan metabolites in first-episode neuroleptic-naive patients with schizophrenia. Mol Psychiatry. 2010;15(9): 938-53. https://doi.org/10.1038/mp.2009.33. 
57. Reichenberg A, Rieckmann N, Harvey PD. Stability in schizophrenia symptoms over time: findings from the Mount Sinai Pilgrim Psychiatric Center Longitudinal Study. J Abnorm Psychol. 2005;114(3):363-72. https:// doi.org/10.1037/0021-843X.114.3.363.

58. Ansell BR, Dwyer DB, Wood SJ, Bora E, Brewer WJ, Proffitt TM, et al.

Divergent effects of first-generation and second-generation antipsychotics

on cortical thickness in first-episode psychosis. Psychol Med. 2015;45(3):515-

27. https://doi.org/10.1017/S0033291714001652.

\section{Publisher's Note}

Springer Nature remains neutral with regard to jurisdictional claims in published maps and institutional affiliations.

Ready to submit your research? Choose BMC and benefit from:

- fast, convenient online submission

- thorough peer review by experienced researchers in your field

- rapid publication on acceptance

- support for research data, including large and complex data types

- gold Open Access which fosters wider collaboration and increased citations

- maximum visibility for your research: over $100 \mathrm{M}$ website views per year

At $\mathrm{BMC}$, research is always in progress.

Learn more biomedcentral.com/submissions 